\title{
Nanoscopic Confinement through Self-Assembly: Crystallization within Micellar Cores Exhibits Simple Gibbs-Thomson Behavior
}

\author{
Thomas Zinn, ${ }^{1}$ Lutz Willner, ${ }^{2}$ and Reidar Lund ${ }^{1, *}$ \\ ${ }^{1}$ Department of Chemistry, University of Oslo, P.O. Box 1033, Blindern, 0315 Oslo, Norway \\ ${ }^{2} J$ ülich Centre for Neutron Science (JCNS) and Institute for Complex Systems (ICS), \\ Forschungszentrum Jülich GmbH, 52425 Jülich, Germany
}

(Received 17 July 2014; revised manuscript received 29 September 2014; published 5 December 2014)

\begin{abstract}
It is well known that liquids confined to small nanoscopic pores and droplets exhibit thermal behavior very different from bulk samples. Less is known about liquids spontaneously confined through selfassembly into micellar structures. Here we demonstrate, using a very well-defined n-alkyl-poly(ethylene oxide) polymer system with a tunable structure, that $n$-alkane(s) forming 2-3 nm small micellar cores are affected considerably by confinement in the form of melting point depressions. Moreover, comparing the reduction in melting points, $\Delta T_{m}$, determined through volumetric and calorimetric methods with the micellar core radius, $R_{c}$, obtained from small-angle x-ray scattering, we find excellent agreement with the well-known Gibbs-Thomson equation, $\Delta T_{m} \sim R_{c}^{-1}$. This demonstrates that the reduced size, i.e., the Laplace pressure, is the dominant parameter governing the melting point depression in micellar systems.
\end{abstract}

The presence of a nanometer confinement has a pronounced effect on the physical properties of liquids, such as phase transition phenomena [1,2]. It is well known that the electronic, mechanical, and thermal behavior of a material in confinement can dramatically differ from its unconfined bulk properties, which is thus of immense importance in nanotechnological applications. Within condensed matter physics, the glass transition and crystallization of liquids confined by nanostructured media such as nanopores $[3,4]$ and nanospheres $[5,6]$ have received strong attention. Probing phase transition phenomena in confined systems is intrinsically difficult due to experimental limitations, structural complexity, etc. [1]. Nevertheless, it has been found that many confined liquids in nanoscopic pore materials, e.g., under hard confinement, exhibit melting depression that seems to depend rather straightforwardly on the domain size [1,2,7-10]. In the absence of specific interactions, the effect of the domain size, $R_{c}$, on the melting point, $T_{m}$, can be expressed by a generalized Gibbs-Thomson equation [11]:

$$
\Delta T=T_{m}^{0}-T_{m}\left(R_{c}\right)=\frac{\alpha}{R_{c}} \frac{\gamma V_{m} T_{m}^{0}}{\Delta H_{\text {fus }}},
$$

where $\gamma$ is the interfacial tension, $T_{m}^{0}$ is the bulk melting temperature of the material, $\Delta H_{\text {fus }}$ is the bulk heat of fusion, $V_{m}$ is the molar volume of the confined material [e.g., $n$-alkane(s)], and $\alpha=3$ for spherical domains. This simple equation seems to hold for a wide range of liquids including $n$-alkane(s) $[12,13]$. However, the relation is neglecting potential complications such as specific, orientational interactions, surface stiffness, surface dynamics, and molecular transport [14]. Whereas in hard confinement the constraining shape is static and fixed in time, materials under soft confinement are characterized by surface fluctuations, deformable geometry, and possibly diffusional processes [15-17]. Examples include block copolymers that selfassemble into various mesoscopic crystal structures (spheres, cylinders, or lamellae) of typically a few tens of nanometer size $[18,19]$. In these systems it has been shown that crystallization can be significantly reduced or even completely suppressed due to surface fluctuations $[14,20]$. Another example of soft confined systems is water in oil emulsion droplets where the size can be controlled by the water content $[17,21]$. Despite the highly dynamic nature of these systems, water was found to crystallize, although the melting temperature was drastically reduced and approximately followed a Gibbs-Thomson dependency. Surface fluctuations in nanometrically confined media are also present in biological systems [22], which are particularly challenging due to their structural and chemical complexity. Micellar systems formed by the self-assembly of amphiphilic molecules can be regarded as generic, simplistic model systems which exhibit many of the same properties as biological systems but are more easily tunable in terms of structure. Here we show that micellar systems formed by n-alkyl-poly(ethylene oxide) $\left(\mathrm{C}_{\mathrm{n}}\right.$-PEO) in water behave as a "self-confining" system analogous to other nanoscopic structures where the melting point depression of the $n$-alkane(s) seems to be directly related to the domain size.

$n$-alkane(s) can be considered one of the simplest chemical structures and have been thoroughly studied in the literature, in particular with respect to the thermodynamical properties [23-25]. Although crystallization in micellar systems is often neglected, there is growing experimental evidence that local crystalline ordering is important and may even drive micelle formation [6,26-28]. It is also known that the melting point in micellar systems is substantially 
reduced. For example, previous studies of commercial surfactants shows that the n-alkyl block partly crystallizes inside the nanometer-sized micellar core $[29,30]$ and that the melting point is substantially reduced in comparison to the pure component [30], although no particular explanation was offered. A substantial reduction of the melting point of $\mathrm{C}_{18}$ chains was also found by using volumetric methods by Sommer et al. on a commercial Brij700 surfactant micelles [31,32]. Recent work on lipid-based polymeric surfactants revealed that the melting of the lipid core was substantially reduced-by about $60^{\circ} \mathrm{C}$. Interestingly, by analyzing the exchange kinetics, an additional activation barrier due to crystallized chains was found below the melting point [33]. Hence crystallization considerably affects the stability of self-assembled structures. While melting point reduction induced by geometrical confinement is commonly observed for simple liquids such as $n$-alkane(s) [8,34-36], it is not clear whether and how confinement effects also play a role in micellar systems.

Here we employ a series of well-defined poly(ethylene oxide)-mono-n-alkyl-ether $\mathrm{C}_{\mathrm{n}}$-PEOx polymers where the size can be varied continuously by varying the length of the n-alkyl blocks, $n=18,21,24,27,28,30$, and PEO molecular weight, $x$, from 5 to $40 \mathrm{~kg} / \mathrm{mol}$. This model system, synthesized by living anionic polymerization, exhibits very low polydispersities, $M_{w} / M_{n} \leq 1.05$, and impurities and is therefore well suited for fundamental investigations. This system spontaneously forms spherical starlike micelles in water where the $2-3 \mathrm{~nm}$ spherical core consists of $n$-alkane(s) that may fully or partially crystallize, surrounded by an extended highly swollen, diffuse PEO polymer shell $[37,38]$. The size of the core, $R_{c}$, can be varied continuously both through the length of the hydrophobic n-alkyl block and the length of the PEO.

The density of the micelles was measured using a high precision Anton Paar, DMA5000 density meter. Assuming incompressibility, the apparent solution density, $d_{\text {polym }}$, can be found by subtracting the pure solvent density $d_{0}$ according to $d_{\text {polym }}=\left(w d_{\text {exp }} d_{0}\right) /\left(d_{0}+(w-1) d_{\text {exp }}\right)$, where $d_{\exp }$ is the measured density of the solution and $w$ is the polymer weight fraction. Figure 1 shows $d_{\text {polym }}$ as a function for both the $\mathrm{C}_{\mathrm{n}}$-PEO5 and $\mathrm{C}_{27}$-PEOx series obtained in $1 \%$ w./w. aqueous solutions. As we have seen, there is a clear indication of discontinuity in solution density upon increasing the temperature which is particularly clear for longer $n$-alkane(s) and, for the $\mathrm{C}_{27}$-PEOx series, for the shorter PEO blocks. This rather sudden decrease in the density is a good indication for a first-order phase transition, i.e., a melting of the $n$-alkane(s) confined in the micellar core. The pure PEO homopolymer $(8 \mathrm{~kg} / \mathrm{mol}$, data not shown) did not exhibit any discontinuity. For the $\mathrm{C}_{27^{-}}$ PEOx series the transitions become progressively weaker at higher PEO lengths, possibly because of the dilution of the n-alkyl signal due to the lower volume fraction. In a work by Sommer et al., who studied commercial Brij700-i.e., $\mathrm{C}_{18}(\mathrm{EO})_{100}$ surfactant micelles-it was found that the density

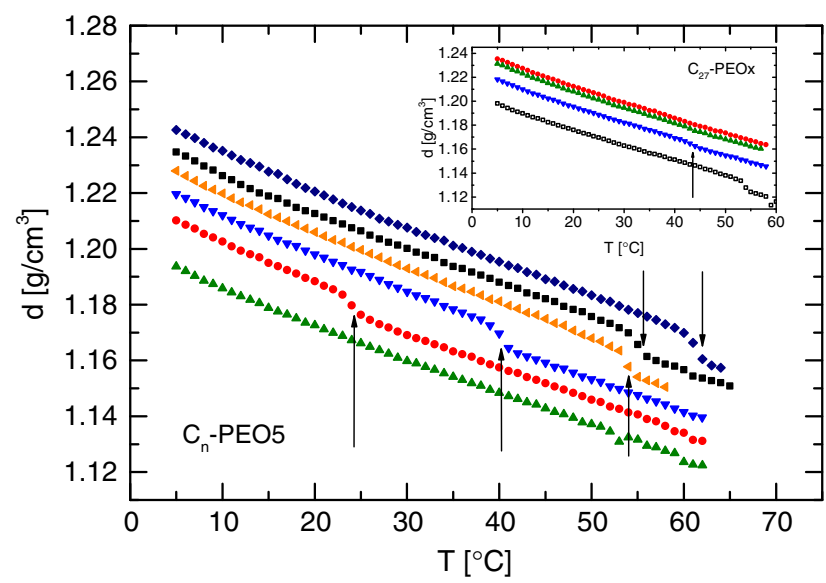

FIG. 1 (color online). Apparent density of $\mathrm{C}_{\mathrm{n}}$-PEO5 for $n=$ $18,21(+0.01), 24(+0.02), 27(+0.03), 28(+0.04), 30(+0.05)$, read from bottom to top (in brackets offset) as a function of temperature in aqueous solution at $1 \%$ polymer weight fraction. The inset contains the apparent densities for $\mathrm{C}_{27}-\mathrm{PEOx}$, $x=5,10,20,40 \mathrm{~kg} / \mathrm{mol}$ (from bottom to top).

continuously decreased [31,32] in a temperature range of between $10^{\circ} \mathrm{C}$ and $90^{\circ} \mathrm{C}$ and there was a hint of discontinuity at the lowest temperature. Although this was not discussed in detail, it could be a signature of a melting transition. In the present case, no melting of the core was detected, even at temperatures down to $5^{\circ} \mathrm{C}$, indicating a drastic reduction of the melting point from $T_{m}=28.2{ }^{\circ} \mathrm{C}$ in bulk [39].

In order to characterize the structure of the micelles in dilute solutions, small-angle x-ray scattering (SAXS) experiments were carried out at different temperatures using an optimized Bruker NanoSTAR instrument (RECX, University of Oslo). In Fig. 2 the scattered intensity, $d \Sigma / d \Omega(Q)$, is plotted as a function of the momentum transfer vector, $Q[Q=4 \pi \sin (\vartheta / 2) / \lambda$, where $\lambda$ is the wavelength and $\vartheta$ the scattering angle], for $1 \%$ solutions of (a) $\mathrm{C}_{18}$-PEO5 and (b) $\mathrm{C}_{28}$-PEO5 at different temperatures. The data show typical features of micellar scattering, with strong scattering at low $Q$ followed by a steep decay at intermediate $Q$, which provides information about the average size of the micelles. At these length scales, we see that the micellar shape and size are not significantly altered with temperature. For the high $Q$ data, which reflects a convoluted scattering from both the core and the polymer corona, we observe an interesting temperature behavior. While for the $\mathrm{C}_{18}$-PEO5 a monotonic increase in the intensity with increasing temperature is visible, $\mathrm{C}_{28}$-PEO5 displays a clear transition from an oscillation pattern with high intensity at high temperatures to a more flat intensity distribution at lower temperatures. This primarily indicates an abrupt change in contrast consistent with a change in electron density upon crossing the melting point. A very similar behavior for the other, higher $n$-alkane(s) is evident at higher temperatures, indeed suggesting that the behavior is caused by the melting of the micellar cores. In order to quantify this behavior, the data were analyzed using a 


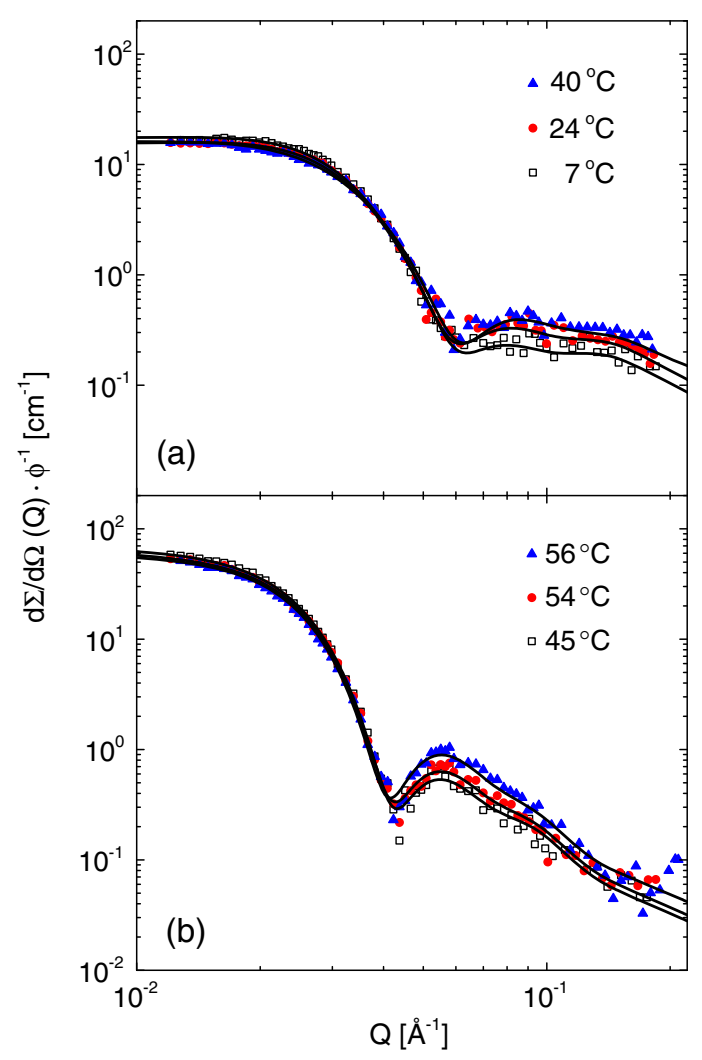

FIG. 2 (color online). SAXS experimental data and fit results for (a) $\mathrm{C}_{18}$-PEO5 and (b) $\mathrm{C}_{28}-$ PEO5 aqueous solutions at $1 \%$ for varying temperatures. Fit curves are shown as solid lines.

detailed core-shell form factor $[37,40]$ that includes interactions within the micellar shell and a graded density distribution. The main parameters were the mass density of the $n$-alkane, micellar radius, $R_{m}$, the aggregation number, $P$, the radius of gyration of PEO, $R_{g}$, and the effective virial coefficient, $\nu$, inside the swollen semidilute corona (for more information, see the Supplemental Material [41]). The temperatures for the SAXS experiments were chosen such that the scattering curves below and above the transition points determined from the densiometry were measured. Since for SAXS the scattering contrast is given by the electron density difference, we explicitly considered the determined apparent densities of $\mathrm{C}_{\mathrm{n}}-\mathrm{PEO} 5$ polymers for modeling the experimental data. The results show that for the description of the discontinuity in the data upon crossing the melting point, an abrupt change in the density of the $n$-alkane(s) had to be considered. As an example, for the $\mathrm{C}_{28}-\mathrm{PEO} 5$ system we find $0.840 \mathrm{~g} / \mathrm{cm}^{3}\left(0.840 \mathrm{~g} / \mathrm{cm}^{3}\right)$ at $54^{\circ} \mathrm{C}$ and $0.824 \mathrm{~g} / \mathrm{cm}^{3}$ $\left(0.797 \mathrm{~g} / \mathrm{cm}^{3}\right)$ just two degrees above, at $56^{\circ} \mathrm{C}$. This compares very nicely with the data obtained through densitometric methods (values given in brackets). For the other polymers we find similar behavior [41]. The structural data and thermodynamical parameters of the pure components are found in Table I. A thermal analysis was performed using a differential scanning calorimeter (DSC) TA Instruments "nano-DSC" instrument for solutions that allows for detecting heat flows on a $\mu \mathrm{J} / \mathrm{s}$ range. The results for the normalized specific heat
TABLE I. Structural and thermal characterization of the $\mathrm{C}_{\mathrm{n}}$-PEOx polymer micelles in $1 \%$ aqueous solutions at $25^{\circ} \mathrm{C}$.

\begin{tabular}{lccccccc}
\hline \hline & $\begin{array}{c}M_{n}^{\text {PEO }} \\
{[\mathrm{kg} / \mathrm{mol}]}\end{array}$ & $P$ & $\begin{array}{c}R_{m} \\
{[\mathrm{~nm}]}\end{array}$ & $\begin{array}{c}R_{c} \\
{[\mathrm{~nm}]}\end{array}$ & $\begin{array}{c}T_{m}^{0 \mathrm{~b}} \\
{\left[{ }^{\circ} \mathrm{C}\right]}\end{array}$ & $\begin{array}{c}\Delta T_{m} \\
{[\mathrm{~K}]}\end{array}$ & $\begin{array}{c}\Delta H_{\text {fus }}^{0}{ }^{\mathrm{b}} \\
{[\mathrm{kg} / \mathrm{mol}]}\end{array}$ \\
\hline 18 & 4.0 & 20 & 8.6 & 1.3 & 28.2 & & 61.3 \\
21 & 4.1 & 48 & 10.1 & 1.9 & 40 & 16.6 & 45.2 \\
24 & 4.2 & 73 & 10.7 & 2.2 & 50.4 & 11.1 & 54.4 \\
27 & 4.2 & 107 & 12.7 & 2.4 & 59.2 & 4.8 & 61.9 \\
& $10.4^{\mathrm{a}}$ & $39^{\mathrm{a}}$ & $14.2^{\mathrm{a}}$ & $1.8^{\mathrm{a}}$ & & 14.9 & \\
& $21.2^{\mathrm{a}}$ & $41^{\mathrm{a}}$ & $20.8^{\mathrm{a}}$ & $1.9^{\mathrm{a}}$ & & 15.8 & \\
& $36.0^{\mathrm{a}}$ & $30^{\mathrm{a}}$ & $28.2^{\mathrm{a}}$ & $1.7^{\mathrm{a}}$ & & 16.2 & \\
28 & 4.6 & 92 & 11.9 & 2.7 & 61.1 & 4.9 & 65.1 \\
30 & 4.3 & 122 & 11.5 & 3.0 & 65.1 & 2.4 & 68.3 \\
\hline \hline
\end{tabular}

${ }^{\mathrm{a}}$ Taken from SANS experiments [38].

${ }^{\mathrm{b}}$ Values for $n$-alkane(s) in bulk taken from Ref. [39].

capacity, $C_{p}$ (heating rate of $2 \mathrm{~K} / \mathrm{min}$ ), are shown in Fig. 3. As we have seen, the specific heat capacity, $C_{p}$, exhibits pronounced peaks that are strongly dependent on the n-alkyl chain length. A close inspection of the data reveals that the peak positions coincide closely with the expected melting points obtained from densiometry and SAXS, again confirming the first-order phase transitions. Again, for the $\mathrm{C}_{18}$, no detection of melting could be made within the measured temperature range. From the area of the peaks we can roughly estimate degree of crystallization, $X_{c}$, from $\Delta H_{\text {fus }}=\int C_{p} d T$ and by comparing these values with the pure $n$-alkane(s) [39]. The analysis suggests an increase of $X_{c} \approx 0.5-0.8$ with increasing nalkyl length. For the $\mathrm{C}_{27}$-PEOx we observe an almost constant $X_{c} \approx 0.6$, independent of the PEO length [41].

Focusing on the data of Fig. 3(b), we see that the melting of $\mathrm{C}_{27}$-PEOx is clearly visible-also for the higher PEO molecular weights. The transition point shifts significantly depending on the PEO chain length and tends to broaden with increasing PEO molecular weight, most markedly from 5 to $10 \mathrm{~kg} / \mathrm{mol}$. Interestingly, the melting point for $\mathrm{C}_{27}$ decreases with increasing $\mathrm{PEO}$ block length. This finding gives a very important insight into the thermodynamic and dynamic factors controlling the phase transition. In particular, is has been found that the molecular exchange kinetics becomes considerably slower upon increasing the PEO length [51]. In other words, while the kinetics becomes slower, the melting point depression is still enhanced. This would suggest that faster exchange kinetics seems not to be directly related to a decrease in the melting point. In order to explain the observed melting point depression, we consider finite size effects. In fact, for the $\mathrm{C}_{27}$-PEOx series we see that the radius of the confining core decreases significantly from 2.6 to $1.7 \mathrm{~nm}$ from PEO of 5 to $40 \mathrm{~kg} / \mathrm{mol}$ as a consequence of increased repulsions between PEO segments in the corona with growing molecular weight, which leads to higher curvature, smaller aggregation number, and, consequently, smaller $R_{c}$ [52]. 

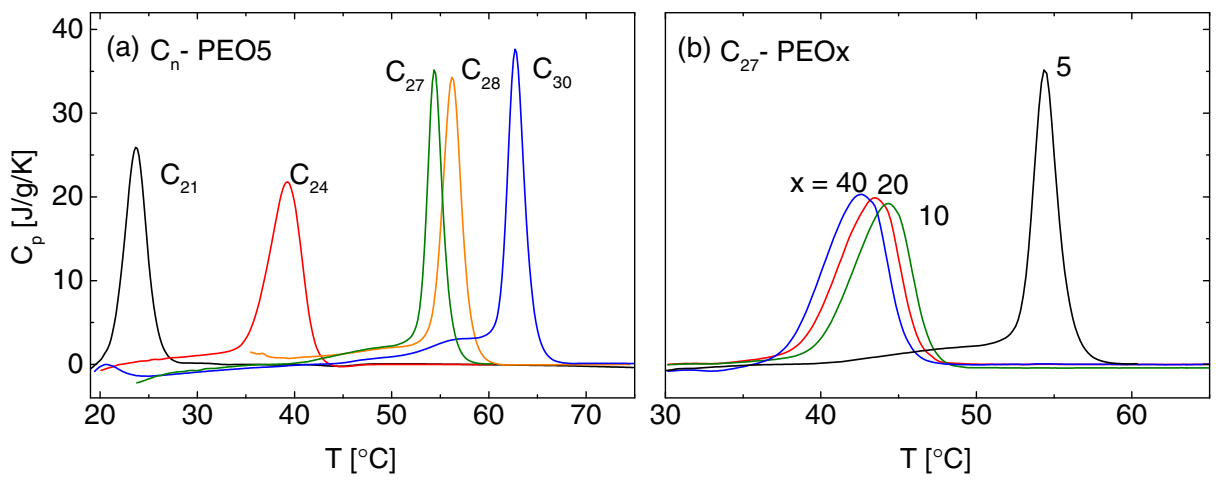

FIG. 3 (color online). nano-DSC thermograms (heating rate: $2 \mathrm{~K} / \mathrm{min}$ ) showing the specific heat capacity $C_{p}$ as a function of temperature for $1 \mathrm{wt} . \%$ solutions of; (a) $\mathrm{C}_{\mathrm{n}}$-PEO5 with various $n$-alkanes $\left(n=21,24,27,28,30\right.$; and (b) $\mathrm{C}_{27}$-PEOx with different PEO lengths $(x=10,20,40 \mathrm{~kg} / \mathrm{mol})$. Baseline is determined from a water reference and is subtracted from the total signal.

In Fig. 4, the melting point depression, $\Delta T_{m}$, is plotted as a function of the inverse of the core radius, $R_{c}^{-1}$, for both $\mathrm{C}_{\mathrm{n}}$-PEO5 and $\mathrm{C}_{27}$-PEOx. As we have seen, the two data sets overlap and display an almost linear behavior in accordance with the Gibbs-Thomson equation. However, in order to reasonably compare the different $n$-alkane(s), the data are rescaled according to Eq. (1), i.e., $\Delta T_{m} \rightarrow$ $\Delta T_{m} /\left(T_{m}^{0} V_{m}\right) \Delta H_{\text {fus }}$, and are replotted in the inset of Fig. 4. Here we have used the experimentally obtained values for $V_{m}$ (at $20^{\circ} \mathrm{C}$ ) and $\Delta H_{\text {fus }}$ taken from the densitometry and DSC, respectively. From the slope of the curve we may extract the effective interfacial tension, $\gamma \approx 6 \mathrm{mN} / \mathrm{m}$. This is lower than what is expected for a pure n-alkane-water interface $(\approx 55-59 \mathrm{mN} / \mathrm{m}$ in the range of $n$-alkane(s) considered [53]). If instead the values for the pure (unconfined) materials $V_{m}$ and $\Delta H_{\text {fus }}$ as assumed in the original Gibbs-Thomson theory are used, we obtain $\gamma \approx 10 \mathrm{mN} / \mathrm{m}$. In any case a lower interfacial tension is evident, which can be rationalized by

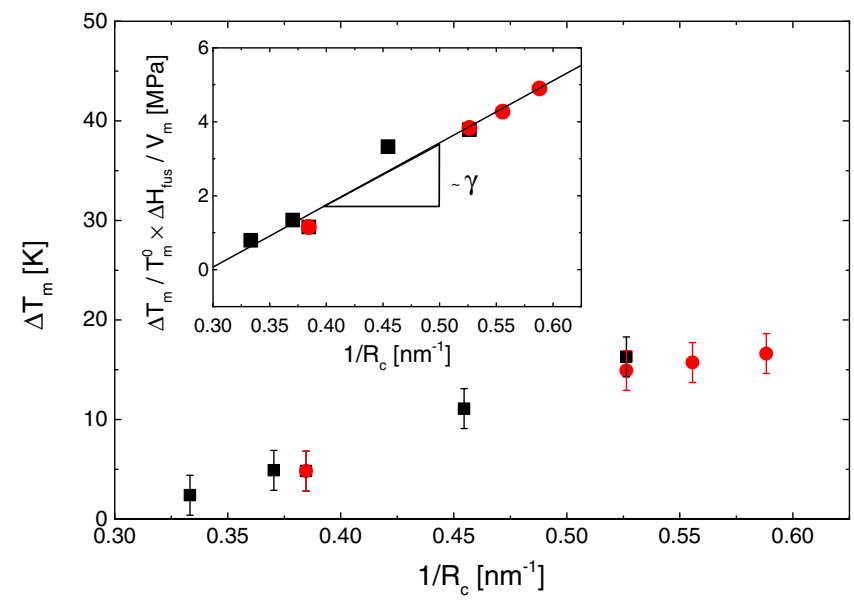

FIG. 4 (color online). Melting point depressions, $\Delta T_{m}$, with respect to the bulk melting point, $T_{m}^{0}$, as a function of the inverse micellar core radius, $R_{c}$, for $\mathrm{C}_{\mathrm{n}}$-PEO5 (square) and $\mathrm{C}_{27}-\mathrm{PEOx}$ (circle) in aqueous solutions of $1 \%$ polymer weight fraction. The error bars are calculated from the full width at half maximum (FWHM) of the DSC curves. The inset shows the same data rescaled, $\Delta T_{m} \rightarrow \Delta T_{m} /\left(T_{m}^{0} V_{m}\right) \Delta H_{\text {fus }}$. the PEO corona that would shield the water molecules from the hydrophobic core surface [54,55].

From Fig. 4 we find an intercept corresponding to $\Delta T_{m}=0$ at $R_{c} \approx 3.4 \mathrm{~nm}$ instead of at $R_{c} \rightarrow \infty$, as expected from the Gibbs-Thomson equation. This apparent inconsistency can be resolved by realizing that the micellar core is not fully crystallized [56]. From the DSC experiments we estimate a fraction of about $0.5-0.8$. Previous studies have attributed this fact to the existence of an amorphous surface layer [1] where the Gibbs-Thomson equation was modified by multiplying Eq. (1) with a term $R_{c} /\left(R_{c}-d\right)$, where $\mathrm{d}$ is the thickness of the layer. Analyzing our data we find a value of about $d \approx 1.2 \mathrm{~nm}$, which is very close to the intrinsic width of the Gaussian core-corona interface obtained from the structural SAXS data, $2 \sigma_{\text {int }}=1-2 \mathrm{~nm}$. Hence it is likely that surface fluctuations induced by the PEO chains on the surface may lead to a disruption of the crystalline domain at the surface. A study by Spehr et al. of the melting point of water confined in small water-in-oil emulsion droplets was also found to be in reasonable agreement with the GibbsThomson equation [17]. Since emulsion droplets are characterized by very small interfacial tensions, the shape fluctuations are expected to be significant and much more pronounced than in the present system. Nevertheless this study and ours both seem to suggest that phase transition can be understood by considering the finite size without evoking fluctuations and molecular dynamics.

In conclusion, micelles spontaneously formed through self-assembly exhibit a melting point depression that appears to be predominantly controlled by the size of the core. This rather surprisingly simple result shows that the melting point transition in micellar systems seems to be dominated by the Laplace pressure regulated by the curvature. The behavior of these self-confining systems can be rather satisfactorily described using thermodynamic arguments and follows essentially the same behavior as hard confined systems, such as liquids in nanopores. These results, which could be obtained only by using a very well-defined micellar system, bring significant insight into the physics of dynamic selfassembled systems and are thus also essential to understanding more complex biological systems. 
We thank Bente A. Breiby (Department of Pharmacy, University of Oslo) for carefully performing the nano-DSC measurements. We gratefully acknowledge grants from the Norwegian Research Council, under the SYNKNOYT program (Grants No. 218411 and No. 228573).

*reidar.lund@kjemi.uio.no

[1] H. K. Christenson, J. Phys. Condens. Matter 13, R95 (2001).

[2] M. Alcoutlabi and G. B. McKenna, J. Phys. Condens. Matter 17, R461 (2005).

[3] D. D. Awschalom and J. Warnock, Phys. Rev. B 35, 6779 (1987).

[4] A. Schreiber, I. Ketelsen, and G. H. Findenegg, Phys. Chem. Chem. Phys. 3, 1185 (2001).

[5] K. Jiang, B. Xie, D. Fu, F. Luo, G. Liu, Y. Su, and D. Wang, J. Phys. Chem. B 114, 1388 (2010).

[6] B. Mai, Z. Li, R. Liu, S. Feng, Q. Wu, G. Liang, H. Gao, and F. Zhu, J. Polym. Res. 20, 299 (2013).

[7] J. Warnock, D. D. Awschalom, and M. W. Shafer, Phys. Rev. Lett. 57, 1753 (1986).

[8] C. L. Jackson and G. B. McKenna, J. Chem. Phys. 93, 9002 (1990).

[9] C. Alba-Simionesco, B. Coasne, and G. Dosseh, J. Phys. Condens. Matter 18, R15 (2006).

[10] B. D. Hamilton, J.-M. Ha, M. A. Hillmyer, and M. D. Ward, Acc. Chem. Res. 45, 414 (2012).

[11] O. Petrov and I. Furó, Phys. Rev. E 73, 011608 (2006).

[12] P. Espeau and J. W. White, J. Chem. Soc., Faraday Trans. 93, 3197 (1997).

[13] S. K. Singh, A. Sinha, G. Deo, and J. K. Singh, J. Phys. Chem. C 113, 7170 (2009).

[14] A. Alegría, R. Lund, F. Barroso-Bujans, A. Arbe, and J. Colmenero, Colloid Polym. Sci. 292, 1863 (2014).

[15] R. Podgornik and V. A. Parsegian, Langmuir 8, 557 (1992).

[16] L. Zhu, B. R. Mimnaugh, Q. Ge, R. P. Quirk, S.Z.D. Cheng, E. L. Thomas, B. Lotz, B. S. Hsiao, F. Yeh, and L. Liu, Polymer 42, 9121 (2001).

[17] T. Spehr, B. Frick, I. Grillo, P. Falus, M. Muller, and B. Stuhn, Phys. Rev. E 79, 031404 (2009).

[18] P. Alexandridis and B. Lindman, Amphiphilic Block Copolymers: Self-Assembly and Applications (Elsevier Science, New York, 2000).

[19] I. W. Hamley, Block Copolymers in Solution: Fundamentals and Applications (John Wiley \& Sons, New York, 2005).

[20] A. V. Tkachenko and Y. Rabin, Phys. Rev. Lett. 76, 2527 (1996).

[21] T. Spehr, B. Frick, I. Grillo, and B. Stühn, J. Phys. Condens. Matter 20, 104204 (2008).

[22] R. Lipowsky and E. Sackmann, Structure and Dynamics of Membranes: I. From Cells to Vesicles/II. Generic and Specific Interactions, edited by R. Lipowsky and E. Sackmann, Handbook of Biological Physics Vol. 1A (Elsevier Science, New York, 1995).

[23] C. M. L. Atkinson and M. J. Richardson, J. Chem. Soc. Faraday Trans. 65, 1749 (1969).

[24] D. H. Bonsor and D. Bloor, J. Mater. Sci. 12, 1552 (1977).

[25] V. Chevallier, M. Bouroukba, D. Petitjean, D. Barth, P. Dupuis, and M. Dirand, J. Chem. Eng. Data 46, 1114 (2001).
[26] L. Yin, T. P. Lodge, and M. A. Hillmyer, Macromolecules 45, 9460 (2012).

[27] J. B. Gilroy, T. Gädt, G. R. Whittell, L. Chabanne, J. M. Mitchels, R. M. Richardson, M. A. Winnik, and I. Manners, Nat. Chem. 2, 566 (2010).

[28] D. Richter, D. Schneiders, M. Monkenbusch, L. Willner, L. J. Fetters, J. S. Huang, M. Lin, K. Mortensen, and B. Farago, Macromolecules 30, 1053 (1997).

[29] F. Heatley, H. H. Teo, and C. Booth, J. Chem. Soc. Faraday Trans. 80, 981 (1984).

[30] P. R. Knowles, R. J. Barlow, F. Heatley, C. Booth, and C. Price, Macromol. Chem. Phys. 195, 2547 (1994).

[31] C. Sommer, J. S. Pedersen, and P. C. Stein, J. Phys. Chem. B 108, 6242 (2004).

[32] C. Sommer, J. S. Pedersen, and V. M. Garamus, Langmuir 21, 2137 (2005).

[33] M. Kastantin, B. Ananthanarayanan, P. Karmali, E. Ruoslahti, and M. Tirrell, Langmuir 25, 7279 (2009).

[34] J. R. Craven, Z. Hao, and C. Booth, J. Chem. Soc., Faraday Trans. 87, 1183 (1991).

[35] J. K. Black, L. E. Tracy, C. P. Roche, P. J. Henry, J. B. Pesavento, and T. Adalsteinsson, J. Phys. Chem. B 114, 4130 (2010).

[36] E. V. Fette, A. Pham, and T. Adalsteinsson, J. Phys. Chem. B 112, 5403 (2008).

[37] T. Zinn, L. Willner, R. Lund, V. Pipich, and D. Richter, Soft Matter 8, 623 (2012).

[38] T. Zinn, L. Willner, R. Lund, V. Pipich, M.-S. Appavou, and D. Richter, Soft Matter 10, 5212 (2014).

[39] W. M. Haynes, CRC Handbook of Chemistry and Physics, 95th ed. (Taylor \& Francis, London, 2014).

[40] C. Svaneborg and J. S. Pedersen, Phys. Rev. E 64, 010802 (2001).

[41] See Supplemental Material at http://link.aps.org/ supplemental/10.1103/PhysRevLett.113.238305, which includes Refs. [42-50], for a detailed description of the experimental methods and SAXS data modeling.

[42] J. S. Pedersen, Macromolecules 36, 416 (2003).

[43] M. Daoud and J. Cotton, J. Phys. (Paris) 43, 531 (1982).

[44] J. S. Pedersen and C. Svaneborg, Curr. Opin. Colloid Interface Sci. 7, 158 (2002).

[45] R. Lund, V. Pipich, L. Willner, A. Radulescu, J. Colmenero, and D. Richter, Soft Matter 7, 1491 (2011).

[46] J. S. Beaucage, J. Appl. Crystallogr. 29, 134 (1996).

[47] K. Devanand and J. C. Selser, Macromolecules 24, 5943 (1991).

[48] M. Wertheim, Phys. Rev. Lett. 10, 321 (1963).

[49] J. S. Pedersen, J. Phys. IV (France) 03, C8-491 (1993).

[50] J. G. Barker and J. S. Pedersen, J. Appl. Crystallogr. 28, 105 (1995).

[51] T. Zinn, Ph.D. thesis, University of Münster [Report No. FZJ-2013-02840, 2013].

[52] C. Tanford, Y. Nozaki, and M. F. Rohde, J. Phys. Chem. 81, 1555 (1977).

[53] A. Goebel and K. Lunkenheimer, Langmuir 13, 369 (1997).

[54] Z. Zhang, G. Xu, F. Wang, and G. Du, J. Dispersion Sci. Technol. 26, 297 (2005).

[55] Y.-Y. Won, H. T. Davis, F. S. Bates, M. Agamalian, and G. D. Wignall, J. Phys. Chem. B 104, 7134 (2000).

[56] F. Font and T. G. Myers, J. Nanopart. Res. 15, 2086 (2013). 